\title{
Stochastic inflation with coloured noise
}

\author{
Heinz-Peter Breuer* and Kerstin E. Kunze ${ }^{\dagger}$ \\ ${ }^{*}$ Physikalisches Institut, Universität Freiburg, Hermann-Herder-Str. 3, D-79104 Freiburg, \\ Germany \\ ${ }^{\dagger}$ Departamento de Física Fundamental, Universidad de Salamanca, Plaza de la Merced s/n, \\ E-37008 Salamanca, Spain
}

\begin{abstract}
In most approaches to stochastic inflation the noise term driving the dynamics is taken to be a white noise process. This white noise is closely linked to a step-function cutoff used to coarse-grain the inflaton field. Taking a different cutoff naturally leads to a coloured noise process. We study the effect of coloured noise on the dynamics of the inflaton, in particular, the resulting probability distributions. Furthermore, the corresponding stochastic differential equation of the inflaton is solved in a perturbative way.
\end{abstract}

Keywords: cosmology, models of inflation

PACS: $98.80 . \mathrm{Cq}$

\section{STOCHASTIC INFLATION}

Infation explains the observed fatness and homogeneity on large scales. Furthermore, during the course of infltion, quantum fluctuations of the inflton are stretched beyond the horizon and turned into classical density fluctuations that serve as seeds of structure formation in the beginning of the epoch of the standard model of cosmology.

Modes leaving the horizon and becoming classical have an effect on the value of the coarse grained inflaton. This backreaction is modelled in the stochastic approach to infltion [1,2]. The effect of quantum fluctuations entering the coarse graining domain, which is larger than the horizon, is modelled by a classical noise term. Thus the evolution of the inflaton is described by a stochastic differential equation.

The nature of the noise term depends on the way of filtering between modes below and above the coarse graining scale. In the original approach, the window function used to filter these modes was taken to be a step function. This led to a white noise in the equation of motion determining the evolution of the coarse grained scalar field [3].

However, it was realized that this is an effect of choosing a sharp cutoff. In general it is expected that the cutoff is smooth rather than sharp. A smooth cutoff leads in general to a coloured noise term in the equation of motion of the inflton (see for example, [4]).

In the following the effect of a coloured noise term will be discussed.

\section{Basic equations}

The dynamics of the inflaton $\phi$ in slow roll infltion is described by the equation

$$
\dot{\phi} \simeq-\frac{V^{\prime}}{3 H},
$$


where $V=V(\phi)$ is the potential and ${ }^{\prime} \equiv \frac{d}{d \phi}$. Furthermore, the Friedmann equation is given by

$$
H^{2} \simeq \frac{8 \pi}{3 M_{P}^{2}} V
$$

In the evolution of the inflaton in its potential there are two competing effects [2]. On the one hand there is the change in the value of $\phi$ due to its classical rolling down the potential. This is given by

$$
\Delta \phi \simeq \dot{\phi} \Delta t
$$

On the other hand, there is the change due to the quantum fluctuations which become classical outside the horizon. These imply a change

$$
\delta \phi= \pm \frac{H}{2 \pi}
$$

During inflation the horizon has a characterisitc size of $H^{-1}$, where $H$ in a de Sitter space-time is strictly constant and in slow roll inflation slowly varies with time. Thus a causal domain has a volume of the order $H^{-3}$. During a time interval $H^{-1}$ there are $e^{3}$ new domains appearing. Each of them contains an almost homogeneous scalar field given by $\phi-\Delta \phi+\delta \phi$. There is a critical value $\phi_{s}$ for which the change due to the quantum fluctuations is larger than the classical change due to the inflaton rolling down its potential. Thus there is the possibility that the infhton "walks up" its potential. This critical value $\phi_{s}$ is determined by $\Delta \phi=\delta \phi$ leading to

$$
\left.\frac{2 \pi}{3} \frac{V^{\prime}}{H^{3}}\right|_{\phi_{s}}=1 .
$$

In a small fraction of domains the field value will continue to grow. However, once the Planck boundary at $V\left(\phi_{M_{P}}\right)=M_{P}^{4}$ is reached inflation will stop. Furthermore, there will be domains in which $\phi$ never becomes small enough in order to stop inftation. Thus there are eternally inflating regions. Of course, our universe evolved from a domain in which inflation stopped at some point. Thus the global structure of the universe as a whole (not just our observable part/universe) becomes very complicated [2]. Our observable universe corresponds only to part of the whole universe.

\section{Effective description of stochastic inflation}

The scalar field is divided into two parts as follows (see, for example, [5]),

$$
\phi(x)=\varphi(x)+\psi(x) .
$$

$\phi$ is the "system field" containing modes whose physical wavelengths are larger than the coarse graining scale, which is larger than the horizon. $\psi$ is the "environment 
field" consisting of field modes whose physical wavelengths are smaller than the coarse graining scale.

The aim is to determine the dynamics of the coarse grained field $\varphi$. This can be written as (see, for example, [6])

$$
\phi(x)=\varphi(x)+\frac{1}{(2 \pi)^{\frac{3}{2}}} \int d^{3} k W(k-\sigma a H)\left[a_{\mathbf{k}} \phi_{\mathbf{k}}(\tau) e^{-i \mathbf{k} \cdot \mathbf{x}}+a_{\mathbf{k}}^{\dagger} \phi_{\mathbf{k}}^{*}(\tau) e^{i \mathbf{k} \cdot \mathbf{x}}\right],
$$

where $W(u)$ is a window function, $\sigma$ a parameter characterizing the size of the coarse graining domain and $a_{\mathbf{k}}$ and $a_{\mathbf{k}}^{\dagger}$ are the usual annihilation and creation operators.

In the original approach to stochastic inflation the window function is chosen to be a step function $W(u)=\theta(u)$ [3]. The equations of motion lead to a stochastic differential equation for the coarse grained field $\varphi(t)$,

$$
\frac{d \varphi}{d t}+\frac{1}{3 H} \frac{d V}{d \varphi}=\frac{H^{\frac{3}{2}}}{2 \pi} \eta(t)
$$

where $\eta(t)$ is a white noise, that is its two-point correlation function is given by

$$
\left\langle\eta(t) \eta\left(t^{\prime}\right)\right\rangle=\delta\left(t-t^{\prime}\right)
$$

According to equation (8) the evolution of $\varphi$ is described by a nonlinear diffusion process. The corresponding probability distribution is determined by a Fokker-Planck equation

$$
\frac{\partial P_{c}}{\partial t}=\frac{\partial}{\partial \varphi}\left(\frac{1}{2} D^{1-\beta} \frac{\partial\left(D^{\beta} P_{c}\right)}{\partial \varphi}+\kappa \frac{d V}{d \varphi} P_{c}\right)
$$

where $D=\frac{H^{3}}{4 \pi^{2}}$ is the diffusion coefficient, $\kappa=\frac{1}{3 H(\varphi)}$ is the mobility coefficient and the parameter $\beta$ determines whether the Itô $(\beta=1)$ or Stratonovich $\left(\beta=\frac{1}{2}\right)$ definition of the stochastic differential equation (8) is used.

The stationary solution $\left(\frac{\partial P_{c}}{\partial t}=0\right)$ of equation (10) is given by

$$
P_{c} \sim \exp \left(\frac{3 M_{P}^{4}}{8 V(\varphi)}\right)
$$

This corresponds to the probability for creation of the universe [7, 2].

The stochastic differential equation (8) can be written more generally as

$$
\frac{d \varphi}{d t}=g(\varphi(t))+\sqrt{D(\varphi(t))} x(t)
$$

where $g$ is the deterministic drift contribution given as before by

$$
g=-\frac{1}{3 H} \frac{d V}{d \varphi} .
$$


Furthermore, $D$ is the diffusion coefficient given by

$$
D(\varphi)=\frac{H^{3}}{4 \pi^{2}}
$$

Finally, $x(t)$ is the noise term. In the case of a step function cutoff we have white noise, $x(t)=\eta(t)$. However, it seems more natural to expect that the cutoff is not exactly sharp, but is a smooth cutoff. Furthermore, white noise is not realized in nature. It is an idealization. The choice of a cutoff different from a step function leads naturally to a coloured noise term. The power spectrum $S(\omega)$ of a given stochastic process is defined by the Fourier transform of its autocorrelation function. Accordingly, the spectrum of a process with a $\delta$-shaped autocorrelation is independent of the frequency $\omega$. This is the reason why it is called white noise. A coloured noise process is characterized by a power spectrum which does depend on frequency [8]. Thus, our aim is to study the stochastic differential equation (12) with a coloured noise term, but leaving the drift term and diffusion coefficient unchanged, that is using equations (13) and (14). Furthermore, it is assumed that the noise process has zero mean, $\langle x(t)\rangle=0$.

\section{COUPLING TO AN ORNSTEIN-UHLENBECK PROCESS}

A Markovian process is a stochastic process $\xi(t)$ with short memory time [9]. To give a precise definition one considers the conditional probability density $p\left(\xi, t \mid \xi_{1}, t_{1} ; \xi_{2}, t_{2} ; \ldots ; \xi_{n}, t_{n}\right)$. This is defined to be the probability density for the process to take the value $\xi$ at time $t$, under the condition that it assumed the values $\xi_{1}, \xi_{2}, \ldots, \xi_{n}$ at corresponding previous times $t_{1}>t_{2}>\ldots>t_{n}$. The process is then said to be Markovian if this conditional probability density only depends on the value $\xi_{1}$ the process assumed at the latest time $t_{1}$, i. e.: $p\left(\xi, t \mid \xi_{1}, t_{1} ; \xi_{2}, t_{2} ; \ldots ; \xi_{n}, t_{n}\right)=p\left(\xi, t \mid \xi_{1}, t_{1}\right)$. This property leads to a great simplification of the mathematical description because it allows to reconstruct the whole hierarchy of joint probability distributions from the conditional density $p\left(\xi, t \mid \xi_{1}, t_{1}\right)$. In the case of a diffusion process, for example, the latter is determined by the Fokker-Planck equation. The mathematical description and analysis of non-Markovian processes is generally much more involved. However, there are certain types of non-Markovian processes that can be described by Markovian processes through the introduction of appropriate auxiliary variables. An example of this type will be discussed in the following.

The time-dependent probability density of a diffusion process is governed by a Fokker-Planck equation (see, for example, equation (10)). A typical example is given by the famous Wiener process. The Wiener process is a Markovian and Gaussian process, but it is not stationary. Another well-known example is the Ornstein-Uhlenbeck process $x(t)$. This process is essentially the only process which is Markovian, Gaussian and stationary. It has an exponentially decaying autocorrelation function

$$
\left\langle x(t) x\left(t^{\prime}\right)\right\rangle=\frac{\sigma^{2}}{2 \gamma} e^{-\gamma\left|t-t^{\prime}\right|},
$$


and obeys the stochastic differential equation

$$
\frac{d}{d t} x(t)=-\gamma x(t)+\sigma \eta(t)
$$

where $\eta(t)$ describes white noise. The quantity $\gamma$ is the relaxation rate and $\tau_{c}=\gamma^{-1}$ represents the autocorrelation time, while $\sigma$ measures the strength of the fluctuations.

The aim is to study the effects of a coloured noise in the equation of motion of the inflaton (cf. equation (12)). Strictly speaking the form of the noise process is determined by the choice of a particular window function in equation (7) which in turn is determined by the coarse graining procedure. The coarse graining procedure is influenced by the model of decoherence of the quantum fuctuations leaving the horizon and becoming classical. However, since, to our knowledge, there is no preferred choice of the window function, we take a phenomenological approach and try to investigate in general the effects of a coloured noise process on the dynamics of the coarse grained scalar field $\varphi$.

The Ornstein-Uhlenbeck process is an example of a noise process that is coloured but still accessible to both the analysis with a Fokker-Planck type equation and direct solution of the corresponding stochastic differential equation. Coupling the infthon to an Ornstein-Uhlenbeck process leads to the following set of stochastic differential equations,

$$
\begin{aligned}
\frac{d}{d t} \varphi(t) & =g(\varphi(t))+\sqrt{D(\varphi(t))} x(t) \\
\frac{d}{d t} x(t) & =-\gamma x(t)+\gamma \eta(t)
\end{aligned}
$$

where the drift coefficient $g$ and diffusion coefficient $D$ are defined as before. In equation (18) the parameter $\sigma$ which measures the strength of the fluctuations of $x(t)$ was taken to be equal to the relaxation rate $\gamma$. This leads to the autocorrelation function

$$
\left\langle x(t) x\left(t^{\prime}\right)\right\rangle=\frac{\gamma}{2} e^{-\gamma\left|t-t^{\prime}\right|},
$$

such that in the limit of zero autocorrelation time, $\tau_{c} \rightarrow 0$, one recovers the $\delta$-function correlation of white noise. The power spectrum of this process is given by

$$
S(\omega)=\int d \tau e^{i \omega \tau}\langle x(t+\tau) x(t)\rangle=\frac{\gamma^{2}}{\omega^{2}+\gamma^{2}} .
$$

Thus, for a finite autocorrelation time the course grained scalar field $\varphi(t)$ follows a nonMarkovian dynamics because it is coupled to a coloured noise process $x(t)$ involving a high-frequency cutoff of the order of the relaxation rate. One might argue that the correlation time $\tau_{c}=\gamma^{-1}$ of the process is of the order of the decoherence time that it takes the quantum modes to become classical. A decoherence time scale is given by the Hubble time, thus one might choose

$$
\gamma=H
$$


With this choice, $\gamma$ becomes a function of time, though slowly varying in slow roll inflation. Equation (18) then describes some kind of generalized Ornstein-Uhlenbeck process, since the process is no longer stationary.

The joint process $(\varphi(t), x(t))$ is Markovian. Therefore, it is possible to write down a Fokker-Planck equation for the corresponding probability distribution $P=P(\varphi, x, t)$, given by [10]

$$
\frac{\partial P(\varphi, x, t)}{\partial t}=-\frac{\partial}{\partial \varphi}[(g+\sqrt{D} x) P]+\frac{\partial}{\partial x}[\gamma x P]+\frac{1}{2} \frac{\partial^{2}}{\partial x^{2}}\left[\gamma^{2} P\right] .
$$

Integrating out the auxiliary variable $x$ gives the reduced probability distribution,

$$
Q(\varphi, t)=\int d x P(\varphi, x, t)
$$

Although the process $\varphi(t)$ is in general non-Markovian one can construct for constant $\gamma$ an effective Fokker-Planck type equation for the reduced probability distribution $Q(\varphi, t)$ [10],

$$
\frac{\partial Q(\varphi, t)}{\partial t}=-\frac{\partial}{\partial \varphi} g Q+\frac{1}{2}\left(1-e^{-\gamma t}\right) \frac{\partial}{\partial \varphi} \sqrt{D} \frac{\partial}{\partial \varphi} \sqrt{D} Q .
$$

The explicit time dependence of the diffusion coefficient is due to the non-Markovian property of the coarse-grained field $\varphi(t)$. Furthermore, for times much larger than the correlation time, $t \gg \gamma^{-1}$, equation (24) determining $Q(\varphi, t)$ approaches the FokkerPlanck equation in the case where the infloton is coupled to a white noise process (cf. equation (10)). Moreover, it is the Fokker-Planck equation (10) in the Stratonovich interpretation.

Compared with the coupling to a white noise process, the dynamics of the probability distribution for the coarse grained field $\varphi$ coupled to an Ornstein-Uhlenbeck process is significantly altered for times shorter than the correlation time. Diffusion of the coarse grained field $\varphi$ is highly suppressed for times shorter than the correlation time.

\section{PERTURBATIVE SOLUTIONS}

The stochastic differential equation (12) depends on the particular choice of the noise process $x(t)$. In the last section a particular choice, namely an Ornstein-Uhlenbeck process has been discussed. It was used that for this particular type of coloured noise it is possible to discuss the probability distribution of the coarse grained field $\varphi(t)$ using a Fokker-Planck type equation. In general, however, it is not possible to find FokkerPlanck type equations for the probability distribution of $\varphi(t)$. For an arbitrary form of the coloured noise process the stochastic differential equation (12) has to be solved. This of course is a difficult endeavour. However, if deviations from the deterministic back ground value are assumed to be small, it is possible to solve the stochastic differential equation for the coarse grained scalar field $\varphi(t)$ by a perturbation expansion.

Assume that the coarse grained field $\varphi(t)$ can be expanded as follows [10],

$$
\varphi(t)=\varphi_{c}(t)+\alpha \varphi_{1}(t)+\alpha^{2} \varphi_{2}(t)+\ldots,
$$


where $\varphi_{c}(t)$ is a solution of the deterministic equation $\dot{\varphi}=g(\varphi) . \alpha$ is a parameter that controlls the order of involvement of the noise term, namely, the term proportional to $\alpha$ corresponds to the linear noise approximation. Using the perturbation expansion (25) in the stochastic differential equation

$$
\frac{d \varphi}{d t}=g(\varphi)+\sqrt{D(\varphi)} \alpha x(t)
$$

leads to a set of stochastic differential equations. Up to second order in $\alpha$ [10]

$$
\begin{aligned}
\frac{d \varphi_{c}}{d t} & =-\frac{M_{P}^{2}}{4 \pi} H^{\prime}\left(\varphi_{c}\right) \\
\frac{d \varphi_{1}}{d t} & =-\frac{M_{P}^{2}}{4 \pi} H^{\prime \prime}\left(\varphi_{c}\right) \varphi_{1}+\frac{H^{\frac{3}{2}}\left(\varphi_{c}\right)}{2 \pi} x(t) \\
\frac{d \varphi_{2}}{d t} & =-\frac{M_{P}^{2}}{4 \pi} H^{\prime \prime}\left(\varphi_{c}\right) \varphi_{2}-\frac{M_{P}^{2}}{8 \pi} H^{\prime \prime \prime}\left(\varphi_{c}\right) \varphi_{1}^{2}+\frac{3}{4 \pi} H^{\frac{1}{2}}\left(\varphi_{c}\right) H^{\prime}\left(\varphi_{c}\right) \varphi_{1} x(t),
\end{aligned}
$$

where $^{\prime}=\frac{d}{d \varphi_{c}}$. The solution for $\varphi_{1}$ is given by

$$
\varphi_{1}(t)=\frac{H^{\prime}\left(\varphi_{c}(t)\right)}{2 \pi} \int_{0}^{t} d \tau \frac{H^{\frac{3}{2}}\left(\varphi_{c}(\tau)\right)}{H^{\prime}\left(\varphi_{c}(\tau)\right)} x(\tau)
$$

assuming $\varphi_{1}(0)=0$. Since $\langle x(t)\rangle=0$, it follows that $\left\langle\varphi_{1}(t)\right\rangle=0$. Furthermore,

$$
\left\langle\varphi_{1}^{2}(t)\right\rangle=\left(\frac{H^{\prime}\left(\varphi_{c}(t)\right)}{2 \pi}\right)^{2} \int_{0}^{t} d \tau \int_{0}^{t} d \sigma \frac{H^{\frac{3}{2}}\left(\varphi_{c}(\tau)\right)}{H^{\prime}\left(\varphi_{c}(\tau)\right)} \frac{H^{\frac{3}{2}}\left(\varphi_{c}(\sigma)\right)}{H^{\prime}\left(\varphi_{c}(\sigma)\right)}\langle x(\tau) x(\sigma)\rangle .
$$

And the expectation value of $\varphi_{2}(t)$ is given by

$$
\begin{aligned}
\left\langle\varphi_{2}(t)\right\rangle & =H^{\prime}\left(\varphi_{c}(t)\right) \int_{0}^{t} d \sigma\left[-\frac{M_{P}^{2}}{8 \pi} \frac{H^{\prime \prime \prime}\left(\varphi_{c}(\sigma)\right)}{H^{\prime}\left(\varphi_{c}(\sigma)\right)}\left\langle\varphi_{1}^{2}(\sigma)\right\rangle\right. \\
& \left.+\frac{3}{8 \pi^{2}} H^{\frac{1}{2}}\left(\varphi_{c}(\sigma)\right) H^{\prime}\left(\varphi_{c}(\sigma)\right) \int_{0}^{\sigma} d \tau \frac{H^{\frac{3}{2}}\left(\varphi_{c}(\tau)\right)}{H^{\prime}\left(\varphi_{c}(\tau)\right)}\langle x(\tau) x(\sigma)\rangle\right] .
\end{aligned}
$$

Thus the autocorrelation function of the noise determines these expectation values. These expressions can be used to calculate the curvature perturbation spectrum resulting in this type of stochastic infhtionary models. This has been done in [10].

\section{CONCLUSIONS}

Replacing the step function cutoff in the original approach to stochastic inflation by any other type of window function leads naturally to a coloured noise term in the equation of motion of the coarse grained scalar field. Here the effect of such a coloured noise was studied in two different settings. For the particular example of coupling 
the inflaton to an Ornstein-Uhlenbeck process it is possible to find a Fokker-Planck type equation for the probability distribution of the coarse grained scalar field. For a general type of coloured noise this is not possible. However, assuming that fluctuations around the deterministic solution of the course grained scalar field remain small, the stochastic differential equation can be solved through a perturbation expansion. This leads to explicit expressions for the dynamics of all statistical quantities in terms of the correlation functions of the coloured noise. In particular, for any given model the procedure yields specific predictions about many quantities of interest, e. g., about the power spectrum, the spectral index and the non-Gaussian character of the fluctuations.

\section{ACKNOWLEDGMENTS}

K.E.K. is supported in part by the programme "Ramón y Cajal" of the M.E.C. (Spain) and Spanish Science Ministry Grants FPA 2002-02037, FPA2005-04823 and BFM 2003-02121.

\section{REFERENCES}

1. A. D. Linde, Phys. Lett. B 175 (1986) 395; H. E. Kandrup, Phys. Rev. D 39 (1989) 2245; A. D. Linde and A. Mezhlumian, Phys. Lett. B 307 (1993) 25;

2. A. D. Linde, D. A. Linde and A. Mezhlumian, Phys. Rev. D 49 (1994) 1783;

3. A. A. Starobinsky in Field Theory, Quantum Gravity and Strings ed. H. J. de Vega and N. Sanchez; A. S. Goncharov and A. D. Linde, Sov. Phys. JETP 65 (1987) 635.

4. S. Winitzki and A. Vilenkin, Phys. Rev. D 61 (2000) 084008.

5. E. Calzetta and B. L. Hu, Phys. Rev. D 52 (1995) 6770.

6. J. Martin and M. A. Musso, Phys. Rev. D 71 (2005) 063514.

7. J. B. Hartle and S. W. Hawking, Phys. Rev. D 28 (1983) 2960.

8. H. Risken, The Fokker-Planck Equation (Springer-Verlag, Heidelberg, Germany, 1996).

9. H. P. Breuer and F. Petruccione, The Theory of Open Quantum Systems (Oxford University Press, Oxford, 2002).

10. H. P. Breuer and K. E. Kunze, in preparation. 